(c) 2010 IEEE. Personal use of this material is permitted. Permission from IEEE must be obtained for all other uses, in any current or future media, including reprinting/republishing this material for advertising or promotional purposes, creating new collective works, for resale or redistribution to servers or lists, or reuse of any copyrighted component of this work in other works. 


\section{Dirty Paper Coding Based Optimal MIMO Relay Communications}

\author{
Muhammad R. A. Khandaker \\ Dept. of Electrical and Computer Engineering \\ Curtin University of Technology \\ Bentley, WA-6102, Australia \\ Email: m.khandaker@postgrad.curtin.edu.au
}

\author{
Yue Rong \\ Dept. of Electrical and Computer Engineering \\ Curtin University of Technology \\ Bentley, WA-6102, Australia \\ Email: y.rong@curtin.edu.au
}

\begin{abstract}
In this paper, we address the optimal source and relay matrices design issue for a multiple-input multiple-output (MIMO) relay network using the dirty paper coding (DPC) scheme at the source node. The aim is to minimize the meansquared error (MSE) of the signal waveform estimation at the destination. Using the property of uplink-downlink duality, the original DPC-based MIMO relay system is first converted to a dual system with a decision feedback equalizer (DFE) at the destination. Then we jointly optimize the source and relay matrices of the dual system. Finally the optimal source and relay matrices of the DPC-based system are obtained by exploiting the link between the source, relay, and destination matrices of the original and dual MIMO relay systems. Simulation results demonstrate that the proposed DPC-based MIMO relay system performs much better than the existing linear minimal MSE (MMSE)-based relaying approach in terms of bit-error-rate.
\end{abstract}

\section{INTRODUCTION}

Relay communication is well known for being a costeffective approach in improving the energy-efficiency of communication system in the case of long source-destination distance. When nodes in the relay network are equipped with multiple antennas, we call such system a multipleinput multiple-output (MIMO) relay system. MIMO relays are particularly useful in extending the network coverage and improve the link reliability of the network.

The capacity of a MIMO relay channel has been studied in [1] and [2]. Several other works studied the optimal relay amplifying matrix for a variety of objective functions. In [3] and [4], the optimal relay amplifying matrix which maximizes the mutual information (MI) between source and destination was derived assuming that the source covariance matrix is an identity matrix. In [5] and [6], minimal mean-squared error (MMSE)-based approaches for MIMO relay systems have been studied. Assuming that a linear MMSE receiver is used at the destination, the structure of the optimal source precoding matrix and the optimal relay amplifying matrices for most commonly used objective functions have been investigated in [7] and [8]. All these works [3]-[8] considered a linear transmitter at the source and a linear receiver at the destination.

A MIMO relay system using a nonlinear decision feedback equalizer (DFE) at the destination has been developed in [9]. The DFE technique is also well-known as the successive interference cancellation (SIC) technique. It is shown in [9] that the DFE-based MIMO relay system has a much better bit-error-rate (BER) and MI performance than MIMO relay systems using linear receivers. However, an inherent drawback of the DFE receiver is the error propagation effect, especially in the case of large modulation constellations and/or large system dimension [10].

An efficient approach to remove the error propagation effect of the DFE receiver is to perform the interference cancellation at the source node. This error propagation-free interference cancellation scheme is known as dirty paper coding (DPC) from Costa's seminal work [11]. The optimal source precoding matrix and the optimal receiving matrix of a single-hop (pointto-point) MIMO system using the DPC scheme have been developed in [12]. It has been shown that in the case of large modulation constellations, the DPC-based MIMO system has better BER performance than that of MIMO system using the DFE receiver. The uplink-downlink duality [13] was applied in [12] to optimize the source precoding matrix and the receiving matrix.

In this paper, we consider a MIMO relay system using the DPC scheme at the source node. We aim at jointly optimizing the source precoding matrix and the relay amplifying matrix to minimize the MSE of signal waveform estimation at the destination. The optimization problem is highly nonconvex and a closed-form solution of the optimal source and relay matrices is intractable. Even the structure of the optimal source and relay matrices of the DPC-based MIMO relay system is difficult to obtain. Thanks to the uplink-downlink duality in MIMO relay channel [14], we first convert the original DPC-based MIMO relay system to a dual system with a DFE receiver at the destination. Then we jointly optimize the source and relay matrices of the dual system by applying the majorization theory [15] and the recently developed matrix generalized triangular decomposition (GTD) tool [9], [16]. Finally the optimal source, relay, and receiver matrices of the DPC-based system are obtained by exploiting the link between the source, relay, and destination matrices of the original and dual MIMO relay system. Simulation results demonstrate that the proposed DPC-based MIMO relay system performs much better than the existing MMSE-based approach in terms of BER.

The rest of this paper is organized as follows. In Section II, 
the system model of a DPC-based MIMO relay system is introduced. The optimal source precoding matrix, the optimal relay amplifying matrix and the optimal receiving matrix are developed in Section III. Section IV shows the simulation results which justify the significance of the proposed DPC-based MIMO relay design. Conclusions are drawn in Section V.

\section{SYSTEM MODEL}

We consider a two-hop MIMO relay communication system as illustrated in Fig. 1 where the source node transmits information to the destination node with the aid of a relay node. The source, relay and destination nodes are equipped with $N_{s}, N_{r}$, and $N_{d}$ antennas, respectively. We denote $N_{b}$ as the number of independent data streams from the source to the destination. To efficiently exploit the system hardware, the relay node uses the same antennas to transmit and receive signals. For simplicity, a linear nonregenerative strategy is applied at the relay node to amplify and forward the received signals.

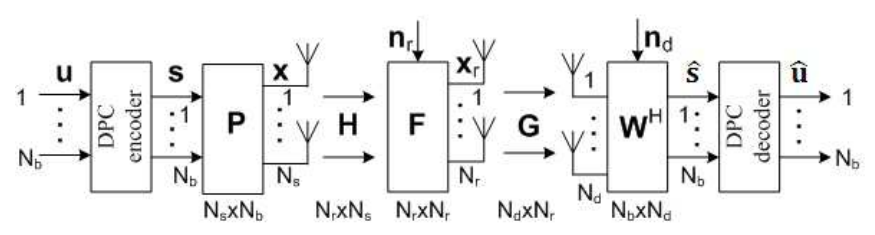

Fig. 1. MIMO relay system model based on DPC.

We make the common assumption that the relay node works in the half-duplex mode. Thus, the communication between the source and destination is completed in two time slots. In the first time slot, a DPC encoder is used at the source node to encode the information-carrying symbol vector $\mathbf{u}$ substreamby-substream removing the interference from the substreams already encoded. We assume that $\mathrm{E}\left[\mathbf{u u}^{H}\right]=\mathbf{I}_{N_{b}}$, where $\mathrm{E}[\cdot]$ stands for the statistical expectation, $(\cdot)^{H}$ denotes the Hermitian transpose, and $\mathbf{I}_{n}$ is an $n \times n$ identity matrix.

Then the $N_{b} \times 1$ DPC-encoded signal vector $\mathbf{s}$ is linearly precoded at the source by the $N_{s} \times N_{b}$ source precoding matrix $\mathbf{P}$. The precoded signal vector

$$
\mathbf{x}=\mathbf{P s}
$$

is transmitted to the relay node from the source node. The received signal vector at the relay node can be written as

$$
\mathbf{y}_{r}=\mathbf{H x}+\mathbf{n}_{r}
$$

where $\mathbf{H}$ is the $N_{r} \times N_{s}$ MIMO channel matrix between the source and the relay, $\mathbf{y}_{r}$ and $\mathbf{n}_{r}$ are the total received signal and the additive Gaussian noise vectors at the relay node, respectively.

In the second time slot, the source node remains silent and the relay node multiplies (linearly precodes) the received signal vector $\mathbf{y}_{r}$ by an $N_{r} \times N_{r}$ relay amplifying matrix $\mathbf{F}$ and transmits the amplified signal vector

$$
\mathbf{x}_{r}=\mathbf{F} \mathbf{y}_{r}
$$

to the destination node. The received signal vector at the destination node can be written as

$$
\mathbf{y}_{d}=\mathbf{G} \mathbf{x}_{r}+\mathbf{n}_{d}
$$

where $\mathbf{G}$ is the $N_{d} \times N_{r}$ MIMO channel matrix between the relay and the destination nodes, $\mathbf{y}_{d}$ and $\mathbf{n}_{d}$ are the total received signal and the additive Gaussian noise vectors at the destination node, respectively. Substituting (1)-(3) into (4), we obtain

$$
\mathbf{y}_{d}=\mathbf{G F H P s}+\mathbf{G F n}_{r}+\mathbf{n}_{d}=\overline{\mathbf{H}} \mathbf{s}+\overline{\mathbf{n}}
$$

where $\overline{\mathbf{H}} \triangleq \mathbf{G F H P}$ is the equivalent MIMO channel matrix of the source-relay-destination link, and $\overline{\mathbf{n}} \triangleq \mathbf{G F} \mathbf{n}_{r}+\mathbf{n}_{d}$ is the equivalent noise vector.

We assume that the channel matrices $\mathbf{H}$ and $\mathbf{G}$ are quasistatic and known to the relay and the destination nodes. The relay node calculates the optimal source $(\mathbf{P})$ and relay $(\mathbf{F})$ matrices and forwards $\mathbf{P}$ and $\overline{\mathbf{H}}$ to the source and the destination nodes, respectively. Without loss of generality, we assume that all noises are independent and identically distributed (i.i.d.) complex circularly symmetric Gaussian noise with zero mean and unit variance. We also assume that a linear receiver is used at the destination node to retrieve the transmitted signals. Thus the estimated signal vector is given by

$$
\hat{\mathbf{s}}=\mathbf{W}^{H} \mathbf{y}_{d}
$$

where $\mathbf{W}$ is an $N_{d} \times N_{b}$ weight matrix. Finally, an estimation of the information-carrying symbol $\hat{\mathbf{u}}$ is obtained by passing $\hat{\mathrm{s}}$ through the DPC decoder.

\section{Optimal DPC-BASED MIMO RELAY SyStem}

In this section, we develop the optimal source precoding matrix $\mathbf{P}$, receiver weight matrix $\mathbf{W}$ and the optimal relay amplifying matrix $\mathbf{F}$ to minimize the MSE of the signal waveform estimation, i.e., $\operatorname{tr}\left(\mathrm{E}\left[(\hat{\mathbf{u}}-\mathbf{u})(\hat{\mathbf{u}}-\mathbf{u})^{H}\right]\right)$, where $\operatorname{tr}(\cdot)$ stands for matrix trace.

\section{A. Uplink-Downlink Duality in MIMO Relay Channel}

A direct construction of the structure of the optimal $\mathbf{P}, \mathbf{F}$ and $\mathbf{W}$ is difficult. In this paper, we exploit the interesting uplink-downlink duality of MIMO relay channel [14] to optimize $\mathbf{P}, \mathbf{F}$ and $\mathbf{W}$. If we treat the DPC-based MIMO relay system in Fig. 1 as a downlink MIMO communication system, then the input-output equation of the associated uplink MIMO relay channel can be written as

$$
\tilde{\mathbf{y}}_{d}=\mathbf{H}^{H} \tilde{\mathbf{F}} \mathbf{G}^{H} \tilde{\mathbf{P}} \mathbf{u}+\mathbf{H}^{H} \tilde{\mathbf{F}} \mathbf{n}_{r}+\tilde{\mathbf{n}}_{d}=\tilde{\mathbf{H}} \mathbf{u}+\tilde{\mathbf{n}}
$$

where $\tilde{\mathbf{y}}_{d}$ and $\tilde{\mathbf{n}}_{d}$ are the $N_{s} \times 1$ total received signal and the Gaussian noise vectors at the receiver of the dual uplink channel, $\tilde{\mathbf{F}}$ is the $N_{r} \times N_{r}$ relay amplifying matrix, $\tilde{\mathbf{P}}$ is the $N_{d} \times N_{b}$ source precoding matrix, $\tilde{\mathbf{H}} \triangleq \mathbf{H}^{H} \tilde{\mathbf{F}} \mathbf{G}^{H} \tilde{\mathbf{P}}$ is the equivalent MIMO channel matrix, and $\tilde{\mathbf{n}} \triangleq \mathbf{H}^{H} \tilde{\mathbf{F}} \mathbf{n}_{r}+\tilde{\mathbf{n}}_{d}$ is the equivalent noise vector in the dual channel. Note that in the dual uplink channel, the roles of the transmitter and the receiver are exchanged. In fact, in the dual system, a linear transmitter is used at the source node, a DFE receiver is 
applied at the destination node, and the channel matrices are replaced by their Hermitian transpose. Moreover, it is shown in [14] that equal signal-to-interference-noise ratios (SINRs) can be achieved in both the uplink and the downlink MIMO relay channel provided that: (1) the order of the symbol detection in the DFE receiver in the dual channel is the reverse of the Costa precoding order in the original channel; (2) the total power allocated at the source and relay node for the dual channel is switched to be the power assigned to the relay and source nodes of the original DPC-based relay channel. Based on this duality property, we can use the receiving filter in the dual uplink channel to construct the source precoding matrix in the DPC-based downlink system, and the receiving matrix in the DPC-based downlink system can be obtained based on the source precoding matrix in the dual uplink channel. Finally, the relay matrix in the downlink system is simply a scaled version of the relay matrix in the dual uplink system. Details will be shown later on in Section III-C.

\section{B. DFE-Based Relay System Design in the Dual Channel}

In the dual channel, a nonlinear DFE receiver is used to detect the information-carrying symbols successively with the $N_{b}$ th symbol detected first and the first symbol detected last. Assuming that there is no error propagation in the DFE receiver, the $k$ th information-carrying symbol is estimated as

$$
\tilde{u}_{k}=\tilde{\mathbf{w}}_{k}^{H} \tilde{\mathbf{y}}_{d}-\sum_{l=k+1}^{N_{b}} c_{k, l} u_{l}, \quad k=1, \cdots, N_{b}
$$

where $\tilde{\mathbf{w}}_{k}$ is the feed-forward vector and $c_{k, l}, l=k+$ $1, \cdots, N_{b}$, are the feedback coefficients for the $k$ th substream. By introducing $\tilde{\mathbf{W}}=\left[\tilde{\mathbf{w}}_{1}, \tilde{\mathbf{w}}_{2}, \cdots, \tilde{\mathbf{w}}_{N_{b}}\right], \tilde{\mathbf{u}}=$ $\left[\tilde{u}_{1}, \tilde{u}_{2}, \cdots, \tilde{u}_{N_{b}}\right]^{T}$, and an $N_{b} \times N_{b}$ strictly upper-triangle matrix $\mathbf{C}$ with nonzero elements $c_{k, l}$, we can represent (5) in matrix form as

$$
\tilde{\mathbf{u}}=\tilde{\mathbf{W}}^{H} \tilde{\mathbf{y}}_{d}-\mathbf{C u}=\left(\tilde{\mathbf{W}}^{H} \tilde{\mathbf{H}}-\mathbf{C}\right) \mathbf{u}+\tilde{\mathbf{W}}^{H} \tilde{\mathbf{n}}
$$

where $\tilde{\mathbf{W}}$ and $\mathbf{C}$ are the feed-forward and feedback matrices of the DFE receiver, respectively. Here $(\cdot)^{T}$ denotes the matrix (vector) transpose. The MSE of the signal waveform estimation of the $k$ th substream, $k=1, \cdots, N_{b}$, is given by

$$
\begin{aligned}
\mathrm{MSE}_{k} \triangleq & \mathrm{E}\left[\left|\tilde{u}_{k}-u_{k}\right|^{2}\right] \\
= & \left|\tilde{\mathbf{w}}_{k}^{H} \tilde{\mathbf{h}}_{k}-1\right|^{2}+\left|\tilde{\mathbf{w}}_{k}^{H} \mathbf{C}_{\tilde{n}} \tilde{\mathbf{w}}_{k}\right|^{2} \\
& +\sum_{l=k+1}^{N_{b}}\left|\tilde{\mathbf{w}}_{k}^{H} \tilde{\mathbf{h}}_{l}-c_{k, l}\right|^{2}+\sum_{l=1}^{k-1}\left|\tilde{\mathbf{w}}_{k}^{H} \tilde{\mathbf{h}}_{l}\right|^{2}
\end{aligned}
$$

where $\mathbf{C}_{\tilde{n}} \triangleq \mathbf{H}^{H} \tilde{\mathbf{F}} \tilde{\mathbf{F}}^{H} \mathbf{H}+\mathbf{I}_{N_{s}}$ is the equivalent noise covariance matrix of the dual channel, and for a matrix $\mathbf{A}, \mathbf{a}_{k}$ is its $k$ th column vector. It is obvious to see from (6) that to minimize the MSEs of the signal estimation, the optimal feedback coefficient should be

$$
c_{k, l}=\tilde{\mathbf{w}}_{k}^{H} \tilde{\mathbf{h}}_{l}, \quad 1 \leq k \leq l \leq N_{b}
$$

or equivalently in matrix form as

$$
\mathbf{C}=\mathcal{U}\left[\tilde{\mathbf{W}}^{H} \tilde{\mathbf{H}}\right]
$$

where $\mathcal{U}\left[\tilde{\mathbf{W}}^{H} \tilde{\mathbf{H}}\right]$ denotes the strictly upper-triangular part of $\tilde{\mathbf{W}}^{H} \tilde{\mathbf{H}}$. Substituting (7) back into (5), we obtain

$$
\tilde{u}_{k}=\tilde{\mathbf{w}}_{k}^{H}\left([\tilde{\mathbf{H}}]_{1: k}[\mathbf{u}]_{1: k}+\tilde{\mathbf{n}}\right), \quad k=1, \cdots, N_{b}
$$

where $[\mathbf{a}]_{1: k}$ denotes a vector containing the first $k$ elements of vector $\mathbf{a}$, and $[\mathbf{A}]_{1: k}$ stands for a matrix containing the first $k$ columns of $\mathbf{A}$.

When the MMSE criterion is used to estimate each symbol, from (8) the feed-forward matrix $\tilde{\mathbf{W}}$ is given as

$$
\tilde{\mathbf{w}}_{k}=\left([\tilde{\mathbf{H}}]_{1: k}[\tilde{\mathbf{H}}]_{1: k}^{H}+\mathbf{C}_{\tilde{n}}\right)^{-1} \tilde{\mathbf{h}}_{k}, \quad k=1, \cdots, N_{b}
$$

where $(\cdot)^{-1}$ denotes the matrix inversion. Let us introduce the following QR decomposition of the augmented matrix

$$
\mathbf{G}_{a} \triangleq\left[\begin{array}{c}
\mathbf{C}_{\tilde{n}}^{-1 / 2} \tilde{\mathbf{H}} \\
\mathbf{I}_{N_{b}}
\end{array}\right]=\mathbf{Q R}=\left[\begin{array}{c}
\overline{\mathbf{Q}} \\
\mathbf{Q}
\end{array}\right] \mathbf{R}
$$

where $\mathbf{R}$ is an $N_{b} \times N_{b}$ upper-triangular matrix with all positive diagonal elements, $\mathbf{Q}$ is an $\left(N_{b}+N_{s}\right) \times N_{b}$ semi-unitary matrix with $\mathbf{Q}^{H} \mathbf{Q}=\mathbf{I}_{N_{b}}, \overline{\mathbf{Q}}$ is a matrix containing the first $N_{s}$ rows of $\mathbf{Q}$, and $\mathbf{Q}$ contains the last $N_{b}$ rows of $\mathbf{Q}$.

Using the QR decomposition (9), it has been shown in [9] that the feed-forward weight matrix $\tilde{\mathbf{W}}$, the feedback matrix $\mathbf{C}$ and the MSE matrix $\mathbf{E}=\mathrm{E}\left[(\tilde{\mathbf{u}}-\mathbf{u})(\tilde{\mathbf{u}}-\mathbf{u})^{H}\right]$ can be written as

$$
\tilde{\mathbf{W}}=\mathbf{C}_{\tilde{n}}^{-1 / 2} \overline{\mathbf{Q}} \mathbf{D}_{R}^{-1}, \quad \mathbf{C}=\mathbf{D}_{R}^{-1} \mathbf{R}-\mathbf{I}_{N_{b}}, \quad \mathbf{E}=\mathbf{D}_{R}^{-2}
$$

where $\mathbf{D}_{R}$ is a matrix taking the diagonal elements of $\mathbf{R}$ as the main diagonal and zero elsewhere. Using (9) and (10), the joint source and relay optimization problem which minimizes the MSE of the signal waveform estimation can be formulated as

$$
\begin{array}{ll}
\min _{\tilde{\mathbf{F}}, \tilde{\mathbf{P}}} & \operatorname{tr}\left(\mathbf{D}_{R}^{-2}\right) \\
\text { s.t. } & {\left[\begin{array}{c}
\mathbf{C}_{\tilde{n}}^{-1 / 2} \tilde{\mathbf{H}} \\
\mathbf{I}_{N_{b}}
\end{array}\right]=\mathbf{Q R}} \\
& \operatorname{tr}\left(\tilde{\mathbf{P}} \tilde{\mathbf{P}}^{H}\right) \leq P_{s} \\
& \operatorname{tr}\left(\tilde{\mathbf{F}}\left[\mathbf{G}^{H} \tilde{\mathbf{P}} \tilde{\mathbf{P}}^{H} \mathbf{G}+\mathbf{I}_{N_{r}}\right] \tilde{\mathbf{F}}^{H}\right) \leq P_{r}
\end{array}
$$

where (13) is the transmit power constraint at the source node, while (14) is the power constraint at the relay node for the dual channel. Here $P_{r}>0$ and $P_{s}>0$ are the corresponding power budgets.

Let $\mathbf{H} \triangleq \mathbf{U}_{H} \boldsymbol{\Sigma}_{H} \mathbf{V}_{H}^{H}$ and $\mathbf{G} \triangleq \mathbf{U}_{G} \boldsymbol{\Sigma}_{G} \mathbf{V}_{G}^{H}$ denote the singular value decomposition (SVD) of $\mathbf{H}$ and $\mathbf{G}$, respectively, where the dimensions of $\mathbf{U}_{H}, \boldsymbol{\Sigma}_{H}, \mathbf{V}_{H}$ are $N_{r} \times N_{r}, N_{r} \times N_{s}$, $N_{s} \times N_{s}$, respectively and the dimensions of $\mathbf{U}_{G}, \boldsymbol{\Sigma}_{G}, \mathbf{V}_{G}$ are $N_{d} \times N_{d}, N_{d} \times N_{r}, N_{r} \times N_{r}$, respectively. We assume that the main diagonal elements of $\boldsymbol{\Sigma}_{H}$ and $\boldsymbol{\Sigma}_{G}$ are arranged in decreasing order. We also introduce $M=\min \left(R_{h}, N_{b}\right)$, where $R_{h} \triangleq \min (\operatorname{rank}(\mathbf{H}), \operatorname{rank}(\mathbf{G}))$ and $\operatorname{rank}(\cdot)$ denotes the rank of a matrix. Using the nonlinear MMSE-DFE receiver at the destination node of the dual channel, the optimal source precoding matrix and the relay amplifying matrix as the solution to the problem (11)-(14) are given by [9]

$$
\tilde{\mathbf{P}}=\mathbf{U}_{G, 1} \boldsymbol{\Lambda}_{P} \mathbf{V}_{P}^{H}, \quad \tilde{\mathbf{F}}=\mathbf{U}_{H, 1} \boldsymbol{\Lambda}_{F} \mathbf{V}_{G, 1}^{H}
$$


where $\boldsymbol{\Lambda}_{P}$ and $\boldsymbol{\Lambda}_{F}$ are $M \times M$ diagonal matrices, $\mathbf{U}_{G, 1}, \mathbf{U}_{H, 1}$ and $\mathbf{V}_{G, 1}$ contain the leftmost $M$ vectors of $\mathbf{U}_{G}, \mathbf{U}_{H}$ and $\mathbf{V}_{G}$, respectively, and $\mathbf{V}_{P}$ is an $N_{b} \times M$ semi-unitary matrix $\left(\mathbf{V}_{P}^{H} \mathbf{V}_{P}=\mathbf{I}_{M}\right)$ such that the QR decomposition in (12) holds. The proof of (15) is similar to the proof of Theorem 2 in [9].

Let us now denote $\boldsymbol{\sigma}_{G_{a}}$ as a column vector containing the singular values of $\mathbf{G}_{a}, \mathbf{d}\left[\mathbf{D}_{R}\right]$ as a column vector containing all diagonal elements of $\mathbf{D}_{R}$, and $\lambda_{p, i}, \sigma_{h, i}, \lambda_{f, i}, \sigma_{g, i}$, $i=1, \cdots, M$, as the $i$ th main diagonal element of $\boldsymbol{\Lambda}_{P}, \boldsymbol{\Sigma}_{H}$, $\boldsymbol{\Lambda}_{F}$, and $\boldsymbol{\Sigma}_{G}$ respectively. Then in [9], it is shown that with the optimal structure of the source and relay matrices (15), the complicated matrix-variable optimization problem (11)-(14) is simplified to an equivalent optimization problem with scalar variables as

$$
\begin{aligned}
& \min _{\left\{\lambda_{p, i}\right\},\left\{\lambda_{f, i}\right\}} \operatorname{tr}\left(\mathbf{D}_{R}^{-2}\right) \\
& \text { s.t. } \quad \mathbf{d}\left[\mathbf{D}_{R}^{2}\right] \prec_{\mathrm{w}}\left[\left\{1+\frac{\left(\lambda_{p, i} \sigma_{h, i} \lambda_{f, i} \sigma_{g, i}\right)^{2}}{\sigma_{h, i}^{2} \lambda_{f, i}^{2}+1}\right\}^{T}, \mathbf{1}_{N_{b}-M}\right]^{T} \\
& \quad \sum_{i=1}^{M} \lambda_{p, i}^{2} \leq P_{s} \\
& \quad \sum_{i=1}^{M} \lambda_{f, i}^{2}\left[\left(\lambda_{p, i} \sigma_{g, i}\right)^{2}+1\right] \leq P_{r} \\
& \quad \lambda_{p, i} \geq 0, \quad \lambda_{f, i} \geq 0, \quad i=1, \cdots, M
\end{aligned}
$$

where $\prec_{\text {w }}$ stands for weakly multiplicative submajorization [15], $\{a\}$ stands for an $M \times 1$ vector $\left[a_{1}, a_{2}, \cdots, a_{M}\right]^{T}$, $\mathbf{1}_{N_{b}-M}$ denotes a $1 \times\left(N_{b}-M\right)$ vector with all 1 elements. Problem (16)-(20) can be efficiently solved using the iterative water-filling approach developed in [9] to obtain $\boldsymbol{\Lambda}_{P}$ and $\boldsymbol{\Lambda}_{F}$. The rotation matrix $\mathbf{V}_{P}$ in (15) can be computed using the matrix GTD developed in [16]. The procedure of optimizing $\tilde{\mathbf{F}}$ and $\tilde{\mathbf{P}}$ of the dual uplink MIMO relay system is summarized in Table I.

TABLE I

Procedure of DFE-BASEd Optimal Relay System Design

1) Compute the SVD of $\mathbf{H}$ and $\mathbf{G}$

2) Obtain $\boldsymbol{\Lambda}_{P}, \boldsymbol{\Lambda}_{F}$ and $\mathbf{D}_{R}$ by solving problem (16)-(20), and obtain $\tilde{\mathbf{F}}=\mathbf{U}_{H, 1} \boldsymbol{\Lambda}_{F} \mathbf{V}_{G, 1}^{H}$.

3) Calculate the GTD

$$
\boldsymbol{\Psi} \triangleq\left[\begin{array}{c}
\mathbf{C}_{\tilde{n}}^{-1 / 2} \mathbf{H}^{H} \tilde{\mathbf{F}} \mathbf{G}^{H} \mathbf{U}_{G, 1} \boldsymbol{\Lambda}_{P} \\
\mathbf{I}_{N_{b}}
\end{array}\right]=\mathbf{Q}_{\Psi} \mathbf{R} \mathbf{P}_{\Psi}^{H}
$$

where $\mathbf{R}$ has same diagonal elements as $\mathbf{D}_{R}$ from step 2 .

4) Obtain the precoding matrix $\tilde{\mathbf{P}}=\mathbf{U}_{G, 1} \boldsymbol{\Lambda}_{P} \mathbf{V}_{P}^{H}$ with $\mathbf{V}_{P}=$ $\left[\mathbf{P}_{\Psi}^{H}\right]_{1: M}$.

5) Compute the feed-forward matrix $\tilde{\mathbf{W}}=\mathbf{C}_{\tilde{n}}^{-1 / 2} \overline{\mathbf{Q}} \mathbf{D}_{R}^{-1}$ and the feedback matrix $\mathbf{C}=\mathbf{D}_{R}^{-1} \mathbf{R}-\mathbf{I}_{N_{b}}$ where $\overline{\mathbf{Q}}$ consists of the first $N_{s}$ rows of $\mathbf{Q}_{\Psi}$

\section{DPC-Based Optimal MIMO Relay System}

Given the optimal source precoding matrix $\tilde{\mathbf{P}}$, relay amplifying matrix $\tilde{\mathbf{F}}$ and receiver matrix $\tilde{\mathbf{W}}$ for the DFE-based relay system design in the dual uplink channel, the precoding matrix for the DPC-based MIMO relay system can be obtained based on theorem 2 in [14] as

$$
\mathbf{P}=\left[\sqrt{\alpha_{1}} \overline{\mathbf{w}}_{1}, \sqrt{\alpha_{2}} \overline{\mathbf{w}}_{2}, \cdots, \sqrt{\alpha_{N_{b}}} \overline{\mathbf{w}}_{N_{b}}\right]
$$

where $\overline{\mathbf{w}}_{k}, k=1, \cdots, N_{b}$, are obtained by scaling $\tilde{\mathbf{w}}_{k}$ in (10) such that $\left\|\overline{\mathbf{w}}_{k}\right\|=1, k=1, \cdots, N_{b}$, and $\alpha_{k}, k=1 \cdots N_{b}$, will be obtained later on in (25) to meet the SINR requirements of all the substreams. Here $\|\cdot\|$ stands for the vector Euclidean norm. The relay amplifying matrix of the DPC-based relay system is $\mathbf{F}=c \tilde{\mathbf{F}}^{H}$ where $c$ is a positive scaling coefficient to meet the power constraint $P_{s}$ at the relay node. The receiving matrix $\mathbf{W}$ is obtained as

$$
\mathbf{W}=\left[\eta_{1} \overline{\mathbf{p}}_{1}, \eta_{2} \overline{\mathbf{p}}_{2}, \cdots, \eta_{N_{b}} \overline{\mathbf{p}}_{N_{b}}\right]
$$

where $\overline{\mathbf{p}}_{k}, k=1, \cdots, N_{b}$ are unit-norm columns of $\tilde{\mathbf{P}}$ in (15), and $\eta_{k}=1 /\left(\sqrt{\alpha_{k}} \overline{\mathbf{p}}_{k}^{H} \mathbf{G F H} \overline{\mathbf{w}}_{k}\right), k=1, \cdots, N_{b}$.

To obtain $\alpha_{k}, k=1, \cdots, N_{b}$, we can assume for the moment that $\eta_{k}=1, k=1, \cdots, N_{b}$, since scaling the receiving vector does not change the output SINR. At the source node, we apply the DPC scheme successively with the first substream encoded first and the last substream encoded last. For the $k$ th substream, the information-carrying symbol $u_{k}$ is encoded into $s_{k}$ by treating $\sum_{l=1}^{k-1} \sqrt{\alpha_{l}} \overline{\mathbf{p}}_{k}^{H} \mathbf{G F H} \overline{\mathbf{w}}_{l} s_{l}$ as the interference known at the transmitter. It should be emphasized that the interference term $\sum_{l=k+1}^{N_{b}} \sqrt{\alpha_{l}} \overline{\mathbf{p}}_{k}^{H} \mathbf{G F H} \overline{\mathbf{w}}_{l} s_{l}$ is unknown at this step. At the receiver side, after applying the linear filter W and the DPC decoder, we obtain $N_{b}$ equivalent subchannels given by

$$
\begin{array}{r}
\hat{u}_{k}=\sqrt{\alpha_{k}} \overline{\mathbf{p}}_{k}^{H} \mathbf{G F H} \overline{\mathbf{w}}_{k} u_{k}+\sum_{l=k+1}^{N_{b}} \sqrt{\alpha_{l}} \overline{\mathbf{p}}_{k}^{H} \mathbf{G F H} \overline{\mathbf{w}}_{l} s_{l}+\overline{\mathbf{p}}_{k}^{H} \overline{\mathbf{n}} \\
k=1, \cdots, N_{b}
\end{array}
$$

with the output SINR

$$
\begin{aligned}
& \beta_{k}=\frac{c^{2}\left|\overline{\mathbf{p}}_{k}^{H} \mathbf{G} \tilde{\mathbf{F}}^{H} \mathbf{H} \overline{\mathbf{w}}_{k}\right|^{2} \alpha_{k}}{\sum_{l=k+1}^{N_{b}} c^{2}\left|\overline{\mathbf{p}}_{k}^{H} \mathbf{G} \tilde{\mathbf{F}}^{H} \mathbf{H} \overline{\mathbf{w}}_{l}\right|^{2} \alpha_{l}+c^{2} \overline{\mathbf{p}}_{k}^{H} \mathbf{G} \tilde{\mathbf{F}}^{H} \tilde{\mathbf{F}} \mathbf{G}^{H} \overline{\mathbf{p}}_{k}+1} \\
& k=1, \cdots, N_{b} \text {. }
\end{aligned}
$$

The power utilized by the relay node in the DPC-based relay system is

$$
\begin{aligned}
P_{s} & =c^{2} \operatorname{tr}\left(\tilde{\mathbf{F}}^{H}\left(\sum_{k=1}^{N_{b}} \alpha_{k} \mathbf{H} \overline{\mathbf{w}}_{k} \overline{\mathbf{w}}_{k}^{H} \mathbf{H}^{H}+\mathbf{I}_{N_{r}}\right) \tilde{\mathbf{F}}\right) \\
& =c^{2}\left(\sum_{k=1}^{N_{b}} \overline{\mathbf{w}}_{k}^{H} \mathbf{H}^{H} \tilde{\mathbf{F}} \tilde{\mathbf{F}}^{H} \mathbf{H} \overline{\mathbf{w}}_{k} \alpha_{k}+\operatorname{tr}\left(\tilde{\mathbf{F}}^{H} \tilde{\mathbf{F}}\right)\right)
\end{aligned}
$$

On the other hand, the SINR in the DFE-based dual channel is given by

$$
\gamma_{k}=\frac{\left|\overline{\mathbf{w}}_{k}^{H} \mathbf{H}^{H} \tilde{\mathbf{F}} \mathbf{G}^{H} \overline{\mathbf{p}}_{k}\right|^{2} p_{k}}{\sum_{l=1}^{k-1}\left|\overline{\mathbf{w}}_{k}^{H} \mathbf{H}^{H} \tilde{\mathbf{F}} \mathbf{G}^{H} \overline{\mathbf{p}}_{l}\right|^{2} p_{l}+\overline{\mathbf{w}}_{k}^{H} \mathbf{H}^{H} \tilde{\mathbf{F}} \tilde{\mathbf{F}}^{H} \mathbf{H} \overline{\mathbf{w}}_{k}+1}
$$

where $p_{k}=\left\|\tilde{\mathbf{p}}_{k}\right\|^{2}, k=1, \cdots, N_{b}$, is the transmit power for the $k$ th substream in the DFE-based relay system. For notational simplicity, let us now denote $a_{k} \triangleq$ 
$\overline{\mathbf{w}}_{k}^{H} \mathbf{H}^{H} \tilde{\mathbf{F}} \tilde{\mathbf{F}}^{H} \mathbf{H} \overline{\mathbf{w}}_{k}, b \triangleq \operatorname{tr}\left(\tilde{\mathbf{F}}^{H} \tilde{\mathbf{F}}\right), d_{k l} \triangleq\left|\overline{\mathbf{p}}_{k}^{H} \mathbf{G} \tilde{\mathbf{F}}^{H} \mathbf{H} \overline{\mathbf{w}}_{l}\right|^{2}$ and $e_{k} \triangleq \overline{\mathbf{p}}_{k}^{H} \mathbf{G} \tilde{\mathbf{F}}^{H} \tilde{\mathbf{F}} \mathbf{G}^{H} \overline{\mathbf{p}}_{k}$. Thus from (22), we have

$$
c^{2}=\frac{P_{s}}{\sum_{k=1}^{N_{b}} a_{k} \alpha_{k}+b} .
$$

Then using (21), (23) and (24), we obtain

$$
\begin{aligned}
& \sum_{l=1}^{k-1} a_{l} \alpha_{l}+\left(a_{k}-\frac{P_{s} d_{k k}}{\gamma_{k}}\right) \alpha_{k}+\sum_{l=k+1}^{N_{b}}\left(d_{k l} P_{s}+a_{l}\right) \alpha_{l} \\
& =-P_{s} e_{k}-b, \quad k=1, \cdots, N_{b}
\end{aligned}
$$

which is a system of $N_{b}$ linear equations and can be easily solved to obtain the values of $\alpha_{k}$. The constant $c$ is then obtained from (24) such that the transmission power at the relay node is constrained to $P_{s}$. It can be shown similar to [14] that the power consumed by the source node is $P_{r}$.

\section{Simulation Results}

In this section, we study the performance of the proposed DPC-based source precoding matrix and relay amplifying matrix through numerical simulations. To precode the $k$ th information-carrying symbol $u_{k}$ into the source symbol $s_{k}, k=1, \cdots, N_{b}$, we apply the Tomlinson-Harashima coding technique [17], [18], which is a simple but suboptimal implementation of the DPC scheme. The Tomlinson-Harashima scheme makes use of modulo operation to remove the interference from the preceding substreams without increasing the transmission power at the source node.

The source, relay and destination nodes are all equipped with multiple antennas. We simulate a flat Rayleigh fading environment where the channel matrices have entries with zero mean and variances $\sigma_{h}^{2} / N_{s}$ and $\sigma_{g}^{2} / N_{r}$ for $\mathbf{H}$ and $\mathbf{G}$, respectively. We define

$$
\mathrm{SNR}_{\mathrm{s}-\mathrm{r}} \triangleq \frac{\sigma_{h}^{2} P_{r} N_{r}}{N_{s}}, \quad \mathrm{SNR}_{\mathrm{r}-\mathrm{d}} \triangleq \frac{\sigma_{g}^{2} P_{s} N_{d}}{N_{r}}
$$

as the signal-to-noise ratio (SNR) of the source-relay and relay-destination links, respectively. All simulation results are averaged over 500 independent channel realizations. We consider two different schemes of SNR in our experiment. First, we vary $\mathrm{SNR}_{\mathrm{S}-\mathrm{r}}$ fixing $\mathrm{SNR}_{\mathrm{r}-\mathrm{d}}=20 \mathrm{~dB}$, then we vary $\mathrm{SNR}_{\mathrm{r}-\mathrm{d}}$ keeping $\mathrm{SNR}_{\mathrm{S}-\mathrm{r}}=20 \mathrm{~dB}$.

We compare the performance of the proposed optimal DPCbased algorithm with the existing MMSE algorithm in [8] and the naive amplify-and-forward (NAF) scheme in terms of BER. For the NAF scheme, we used $\mathbf{P}=\sqrt{\frac{P_{r}}{N_{s}}} \mathbf{I}_{N_{s}}$ and

$$
\mathbf{F}=\sqrt{\frac{P_{s}}{\operatorname{tr}\left(\mathbf{H P P}^{H} \mathbf{H}^{H}+\mathbf{I}_{N_{r}}\right)}} \mathbf{I}_{N_{r}} .
$$

In the simulations, we set $N_{b}=N_{s}=N_{r}=N_{d}=3$. Fig. 2 shows the BER performance of all three algorithms with varying $\mathrm{SNR}_{\mathrm{s}-\mathrm{r}}$ and $\mathrm{SNR}_{\mathrm{r}-\mathrm{d}}=20 \mathrm{~dB}$, whereas Fig. 3 demonstrates the BER performance versus $\mathrm{SNR}_{\mathrm{r}-\mathrm{d}}$ with $\mathrm{SNR}_{\mathrm{s}-\mathrm{r}}=20 \mathrm{~dB}$.

Our results clearly demonstrate the better performance of the proposed DPC-based joint source and relay optimization

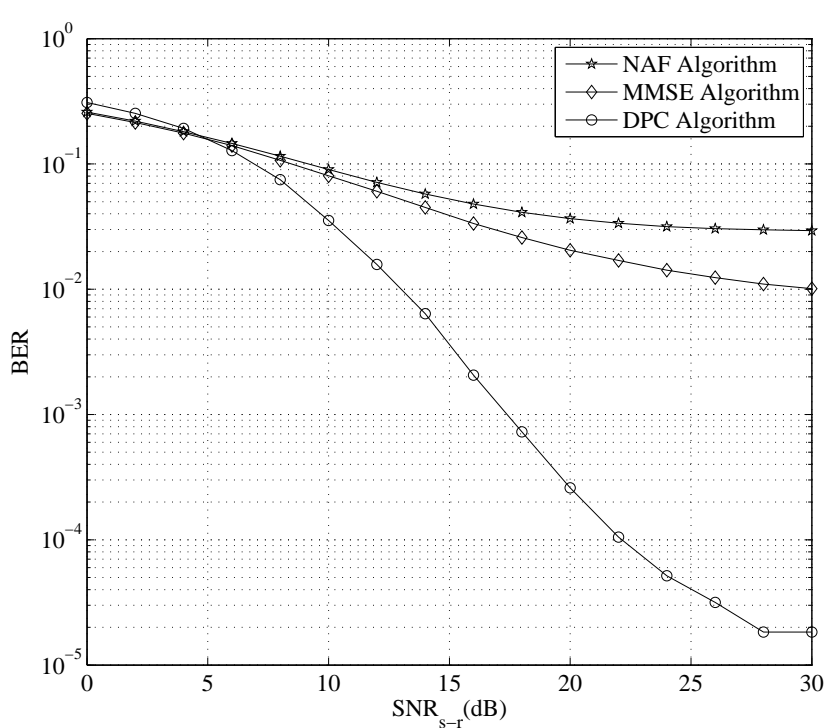

Fig. 2. BER versus $\mathrm{SNR}_{\mathrm{S}-\mathrm{r}}$ in MIMO relay channel. $\mathrm{SNR}_{\mathrm{r}-\mathrm{d}}=20 \mathrm{~dB}$.

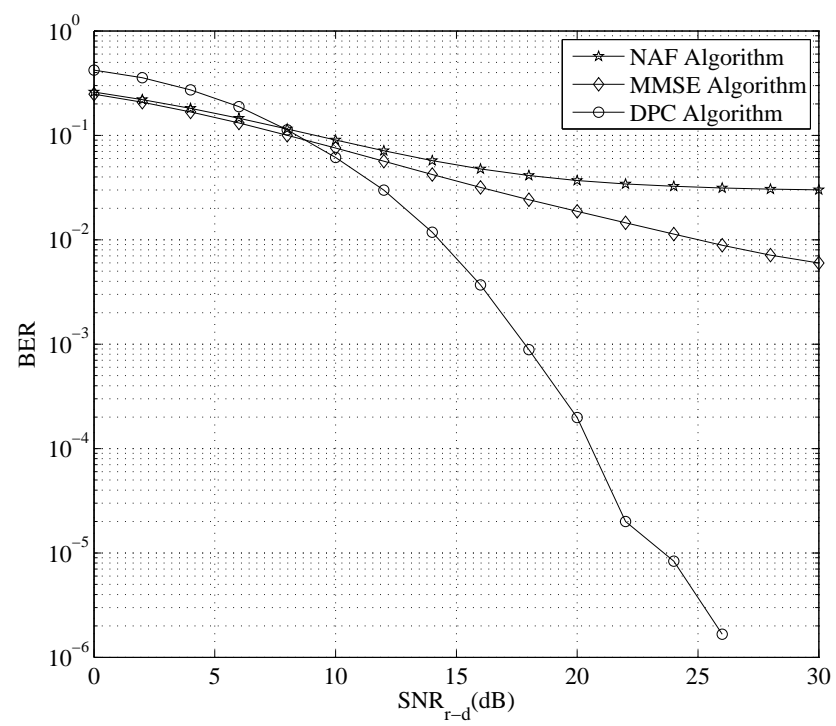

Fig. 3. BER versus $\mathrm{SNR}_{\mathrm{r}-\mathrm{d}}$ in MIMO relay channel. $\mathrm{SNR}_{\mathrm{S}-\mathrm{r}}=20 \mathrm{~dB}$.

technique. It can be seen that the proposed optimal algorithm yields the lowest BER compared to the other two approaches. The existing relay algorithms using a linear receiver yield much higher BER compared with the DPC-based relay technique.

\section{Conclusions}

We have developed the optimal structure of the source precoding matrix and the relay amplifying matrix for nonregenerative DPC-based MIMO relay networks to jointly minimize the MSE of the signal waveform estimation. The uplinkdownlink duality has been applied to optimize the source and 
relay matrices. Simulation results demonstrate that the proposed DPC-based source and relay design algorithm performs much better than the existing linear MMSE-based approach in terms of BER. Future works may include considering DPC for multiuser MIMO relay networks.

\section{REFERENCES}

[1] B. Rankov and A. Wittneben, "On the capacity of relay-assisted wireless MIMO channels," in Proc. 5th IEEE Workshop on Signal Processing Advances in Wireless Commun., Lisbon, Portugal, Jul. 2004, pp. 323327.

[2] B. Wang, J. Zhang, and A. Høst-Madsen, "On the capacity of MIMO relay channels," IEEE Trans. Inf. Theory, vol. 51, pp. 29-43, Jan. 2005.

[3] X. Tang and Y. Hua, "Optimal design of non-regenerative MIMO wireless relays," IEEE Trans. Wireless Commun., vol. 6, pp. 1398-1407, Apr. 2007.

[4] O. Muñoz-Medina, J. Vidal, and A. Agustín, "Linear transceiver design in nonregenerative relays with channel state information," IEEE Trans. Signal Process., vol. 55, pp. 2593-2604, Jun. 2007.

[5] W. Guan and H. Luo, "Joint MMSE transceiver design in nonregenerative MIMO relay systems," IEEE Commun. Lett., vol. 12, pp. 517-519, Jul. 2008.

[6] A. S. Behbahani, R. Merched, and A. M. Eltawil, "Optimizations of a MIMO relay network," IEEE Trans. Signal Process., vol. 56, pp. 50625073, Oct. 2008.

[7] Y. Rong and Y. Hua, "Optimality of diagonalization of multihop MIMO relays," IEEE Trans. Wireless Commun., vol. 8, pp. 6068-6077, Dec. 2009.
[8] Y. Rong, X. Tang, and Y. Hua, "A unified framework for optimizing linear non-regenerative multicarrier MIMO relay communication systems,' IEEE Trans. Signal Process., vol. 57, pp. 4837-4851, Dec. 2009.

[9] Y. Rong, "Optimal linear non-regenerative multi-hop MIMO relays with MMSE-DFE receiver at the destination," IEEE Trans. Wireless Commun., vol. 9, pp. 2268-2279, Jul. 2010.

[10] D. Tse and P. Viswanath, Fundamentals of Wireless Communication. Cambridge University Press, 2005.

[11] M. H. M. Costa, "Writing on dirty paper," IEEE Trans. Inf. Theory, vol. 29, pp. 439-441, May 1983.

[12] D. P. Palomar and Y. Jiang, MIMO Transceiver Design via Majorization Theory. Now Publishers, 2007.

[13] P. Viswanath and D. N. C. Tse, "Sum capacity of the vector Gaussian broadcast channel and uplink-downlink duality," IEEE Trans. Inf. Theory, vol. 49, pp. 1912-1921, Aug. 2003.

[14] K. S. Gomadam and S. A. Jafar, "Duality of MIMO multiple access channel and broadcast channel with amplify-and-forward relays," IEEE Trans. Commun., vol. 58, pp. 211-217, Jan. 2010.

[15] A. W. Marshall and I. Olkin, Inequalities: Theory of Majorization and Its Applications. Academic Press, 1979.

[16] Y. Jiang, W. W. Hager, and J. Li, "The generalized triangular decomposition," Mathematics of Computation, vol. 77, pp. 1037-1056, Apr. 2008.

[17] M. Tomlinson, "New automatic equaliser employing modulo arithmetic," Electronics Lett., vol. 7, pp. 138-139, Mar. 1971.

[18] H. Harashima and H. Miyakawa, "Matched-transmission technique for channels with intersymbol interference," IEEE Trans. Commun., vol. 20 pp. 774-780, Aug. 1972. 